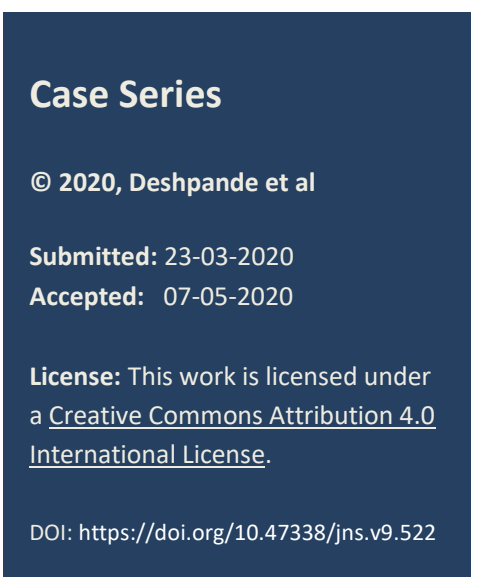

\section{In-utero segmental volvulus around vitellointestinal duct remnants leading to ileal atresia and stenosis: A case series}

Vidyanand Deshpande, ${ }^{*}$ Rajgopal Totla, Arjun Pawar, Pravin Suryawanshi

Department of Surgery, Pediatric Surgery Division, MGM Medical College, Aurangabad

Correspondence*: Vidyanand Deshpande, Department of Surgery, Pediatric Surgery Division, MGM Medical College, Aurangabad, India. E-mail: drvidyananddeshpande@gmail.com

\section{KEYWORDS \\ Volvulus \\ Ileal atresia \\ Ileal stenosis \\ Vitellointestinal duct \\ Meckel's diverticulum}

\section{ABSTRACT}

Background: The etiology of intestinal atresia is multifactorial with vascular accidents being the most common event in its development. In-utero volvulus is a well-known type of vascular accident leading to intestinal atresia. Segmental volvulus around vitellointestinal duct remnants is rarely described vascular accident leading to ileal atresia.

Case Series: We report two cases of segmental ileal volvulus around vitellointestinal duct remnants in neonates leading to ileal atresia in one case and intestinal stenosis in the other one. Both neonates presented with signs of neonatal intestinal obstruction. X-ray abdomen performed in both neonates showed air fluid levels. Both neonates were operated with a suspicion of intestinal atresia. At surgery, a segment of the small bowel was found twisted around vitellointestinal duct remnants in both cases. First case also had intestinal atresia whereas the second one had intestinal stenosis secondary to the segmental volvulus.

Conclusion: Segmental volvulus around vitellointestinal duct remnants can also cause ileal atresia or stenosis.

\section{INTRODUCTION}

Vascular accidents leading to ischemic necrosis and subsequent resorption of the affected segment of the ileum are thought to be predisposing factors in the majority of cases of ileal atresia.[1] Segmental volvulus is the twisting of a bowel loop, around a fixed structure, along the axis of its mesentery.[2] Intrauterine segmental volvulus occasionally occurs around vitellointestinal duct (VID) remnants. Herein, we present two such cases of segmental volvulus around VID remnants in neonates, both being diagnosed as ileal atresia pre-operatively.

\section{CASE SERIES}

Case 1: A 3-day-old, 2.3kg, female neonate was brought with abdominal distension and bilious vomiting since day 1 of life. No antenatal scans were available. On clinical examination, the abdomen was soft and mildly distended. The abdominal radiograph showed multiple air-fluid levels with no pelvic gas shadow. Laboratory investigations were within normal ranges. On exploration, a fibrous cord was seen extending from ileum to umbilicus. The central part of this VID remnant was patent, thinned out, and filled with mucous. This remnant formed an axis around which there were 3 rotations of the adjacent ileal loop. After untwisting the bowel, type II ileal atresia was noted close to the attachment of ileum to the vitellointestinal duct remnant. (Fig.1A,1B).

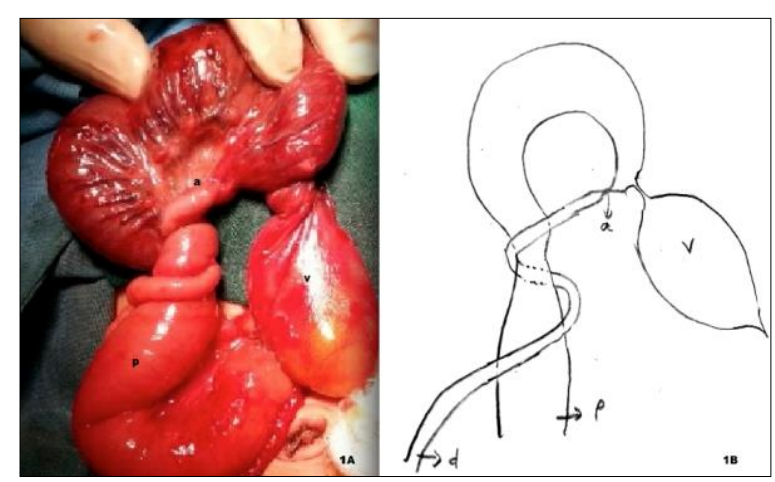

Figure 1A: Segmental volvulus of ileum around the axis of mucous filled vitellointestinal duct remnant (v). Site of atresia (a) is close to attachment of ileum and remnant. Proximal dilated $(\mathrm{p})$ and distal collapsed (d) ileal segments are shown. 1B - Line diagram showing the same findings. a-type II atresia.

Resection and end to back ileal anastomosis were performed. Postoperative recovery was uneventful. Nasogastric tube feeds were started on the 6th day and 
gradually increased to full feeds by the 9th day. The baby was discharged on the 10th day and is doing well in subsequent follow-ups.

Case 2: A full-term, vaginally delivered, 4-day-old male baby $(2.6 \mathrm{~kg}$ weight) presented with bilious vomiting and abdominal distension since day 1 of life. His antenatal scan at 32 weeks of gestation was normal. Clinical examination revealed distension of the abdomen with no other significant findings. An abdominal radiograph was suggestive of intestinal atresia. A provisional diagnosis of intestinal atresia was made. Laboratory investigations were within normal ranges. After initial resuscitation, surgery was planned. Laparotomy revealed segmental volvulus of the bowel around patent vitellointestinal duct (PVID) with segmental stenosis and luminal narrowing of the compressed part of the ileum (Fig.2). Resection of PVID with stenosed segment of ileum was done. End to end ileo-ileal anastomosis was performed. Postoperatively recovery was uneventful. Nasogastric tube feeds were started on the 5 th day and gradually increased to full feeds by the 8 th day. The baby was discharged on the 11 th day and had gained adequate weight in the follow-up visits.

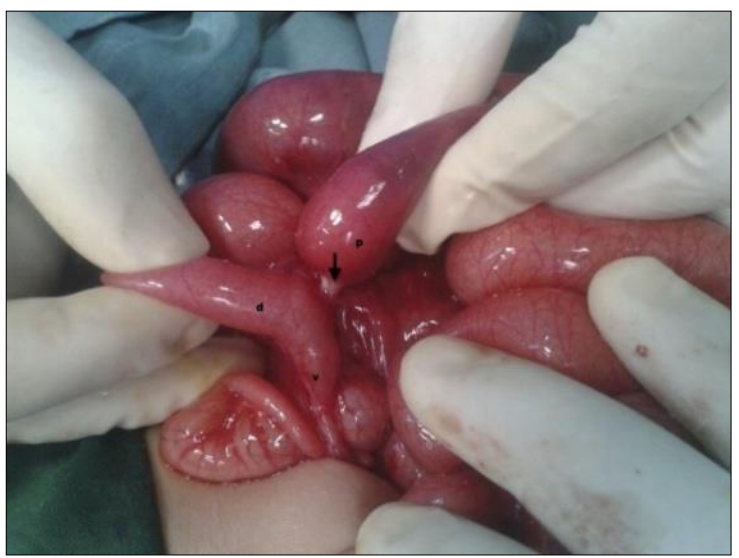

Figure 2: Showing compression and stricture of ileum (arrow) after untwisting of volvulus. Proximal segment (p), distal segment (d) and patent vitellointestinal duct (v) is seen.

\section{DISCUSSION}

Vascular accident is the most accepted theory for ileal atresia.[1] Causes of these vascular accidents can be volvulus, intussusceptions, perforations, thromboembolism, internal hernias, and tight anterior abdominal wall defects. Other known causes of bowel atresia include maternal exposure to certain drugs, cocaine abuse, and smoking.[3]

Neonatal volvulus is a life-threatening condition. It is usually secondary to malrotation. Few studies suggest intestinal volvulus without malrotation occurs in 19$26 \%$ of the small bowel volvulus in children. The etiology remains unknown. However, some of these have predisposing factors such as Meckel's diverticulum, duplication cyst, and meconium plug.[4]

Primary segmental volvulus can also lead to ileal atresia.[2] Secondary intussusceptions with Meckel's diverticulum as a lead point has also been described as a cause of jejunoileal atresia.[5] However, in-utero segmental volvulus around PVID leading to ileal atresia has not been described.

Komuro et al. retrospectively studied intra-operative findings of 48 cases and concluded that volvulus can be the cause as well as the effect of jejunoileal atresia.[6] In another study of 65 cases of jejunoileal atresia by Sinha and Sarin, they found that $16.9 \%$ of cases had volvulus as associated findings.[7]

Just like other causes of intrauterine mesenteric vascular accidents, antenatal volvulus around remnants of the vitellointestinal tract can also cause vascular insufficiency. This can lead to a spectrum of damage to the ileum, with extrinsic compression on one end (case 2 ), and ileal atresia at the site of maximum vascular compromise on the other end (case 1). The severity of damage may depend on the time of volvulus, with antenatal volvulus leading to ileal atresia and perinatal or postnatal volvulus leading to ileal stricture or compression. These two cases represent a part of that spectrum. Here, in case 1 , it is likely that the point of maximum vascular compromise was at the apex of volvulus leading to atresia.

Presentation in both the cases was just like any other case of ileal atresia and hence diagnosis was based on per-operative findings. However antenatal and pre-operative diagnosis is also possible. Prompt surgical intervention in form of resection and ileo-ileal anastomosis leads to a good outcome.

\section{CONCLUSION}

Segmental volvulus around vitellointestinal duct remnants can also be one of the etiologies of ileal atresia and stenosis. Prompt identification and management can lead to a good outcome.

Acknowledgements: Nil

Conflict of Interest: None declared

Source of Support: Nil

Consent to Publication: Author(s) declared taking informed written consent for the publication of clinical photographs/material (if any used), from the legal guardian of the patient with an understanding that every effort will be made to conceal the identity of the patient, however it cannot be guaranteed.

Author Contributions: Author(s) declared to fulfill authorship criteria as devised by ICMJE and approved the final version. 


\section{REFERENCES}

1. Ashcraft KW, Holcomb GW, Murphy JP. Intestinal atresia and stenosis. PediatrSurgInt. 2005; 4th Edition Philadelphia, Pa: Elsevier-Saunders.

2. Park WH, Park SM, Choi SO. Ileal atresia secondary to intrauterine segmental volvulus. J Korean Assoc PaediatricSurg. 1995;1:177-80

3. Werler MM, Sheehan JE, Mitchell AA. Maternal medication use and risks of gastroschisis and small intestinal atresia. Am J Epidemiol. 2002; 155:26-31.

4. Kitano Y, Hashizume K, Okhura M. Segmental small bowel volvulus not associated with malrotation in childhood. Pediatr Surg Int. 1995; 10:335-8.
5. Senocak ME, Buyukpamukcu N, Hicsonmez A. Ileal atresia due to intrauterine intussusception caused by Meckel's diverticulum. Pediatr Surg Int. 1990; 5:64-6.

6. Komuro H, Hori T, Amagai T, Hirai M, Yotsumoto K, Urita Y, et al. The etiologic role of intrauterine volvulus and intussusception in jejunoileal atresia. $\mathrm{J}$ Pediatr Surg. 2004; 39:1812-4.

7. Sinha S, Sarin YK. Outcome of jejuno-ileal atresia associated with intraoperative finding of volvulus of small bowel. J Neonatal Surg. 2012;1:37. 